ON A

\title{
CASE OF AORTIC ANEURISM,
}

IN WHICH

A COMMUNICATION WITH THE PULMONARY ARTERY WAS RECOGNISED DURING LIFE BY MEANS OF

PHYSICAL DIAGNOSIS.

BY

WILLOUGHBY FRANCIS WADE, M.B., M.R.C.P., SENIOR PHYSICIAN TO THE RUEEN'S HOSPITAL, BIRMINGHAM.

COMMUNICATED BY

DR. MARKHAM.

Received May 1st.--Read June 11th, 1861.

The best memoir extant (Dr. Thurnam's) upon spontaneous varicose aneurisms of the aorta is contained in the 'Transactions' of this Society. To it, therefore, the present communication may be most suitably addressed, since my object is to render more precise the physical diagnosis of this disease.

James Selwood, æt. 35, married, a porter in the Parcels Office, at the New Street Railway Station, applied at the Birmingham General Dispensary, on May 1st, 1860. He was a fine-looking, well-made man, and complained of slight cough, some general debility, and a little loss of flesh. These symptoms had been coming on for several months. Suspecting phthisis, I stripped him to examine the lungs. Not finding any sign of disease in the anterior portions of these organs, 
I applied the stethoscope to the heart, and directly heard sounds which convinced me that the case was not one of ordinary valvular disease. I therefore recommended the patient to enter the Queen's Hospital, under my care, which he did in a few days. I found that for four years he had suffered much from piles, and about November, 1859, had lost as much as a pint of blood in a day, and, ever since, the losses have been considerable. To these fluxes he attributed the debility and wasting which induced him to consult me. About Christmas, 1859, his duties, in calling over the parcels, \&c., being at that time very onerous, he suffered for a day or two from hoarseness, and has since often felt a rising in the throat. Two weeks before I first saw him, whilst wheeling a heavy truck across the line, an engine ran up, and to avoid being knocked down, he was obliged to make a violent and sudden spring; he directly felt very faint, and was compelled to sit down for some time, but thought no more about it. He had never had any palpitation. His appetite was good, and he slept well. He had a little cough, with expectoration of watery mucus, and slight dyspnœa on exertion; but the cough was most troublesome on lying down.

Physical examination showed that the cardiac dulness was increased in the vertical direction. The apex could be distinctly seen and felt beating in the sixth intercostal space, and the heart was also to be seen beating in the fifth. Over the cartilage of the fourth left rib two loud murmurs were heard, instead of the usual cardiac sounds; that, with the second sound, being of a hissing character, and so prolonged us to continue till the commencement of the next ventricular systole. At this same spot a very considerable purring tremor accompanied the second murmur. The first murmur was of a loud bellows character. Both murmurs were audible as high as the bifurcation of the common carotids, in the back, and over all the upper part of the chest; they did not seem to be peculiarly propagated towards the left subclavicular space. At the apex of the heart a single murmur only was to be heard, and this evidently attended, or rather re- 
placed, the cardiac first sound; it could be traced easily down to the ensiform cartilage. At the apex, the cardiac second sound was very distinct and quite natural; no trace of murmur.

I found no venous distension on pulse. The pulsation of the carotids was very visible, particularly on the left side and marked by some, though not considerable, thrill. The heart's action was quiet and regular.

The only abnormal physical sign in the lungs was some mucous râles at the base of each, equally on either side. Pupils contracted, but mobile; liver enlarged, no icterus; urine normal.

From this combination of physical signs, I concluded-

1st. That blood escaped either from the aorta or the pulmonary artery during their systole, from the loud hissing, prolonged murmur replacing the second sound at the base of the heart.

2nd. That it was probably from the aorta that the blood escaped, from the propagation of the sound up the arteries of the neck, and their visible pulsation and sensible thrill.

3rd. That the blood did not regurgitate into either ventricle, from the absence of any regurgitant murmur at the apex of the heart, where, on the contrary, an ordinary second sound was audible. It is true that slight regurgitant murmur is not necessarily conveyed to the apex of the heart ; but I have never yet found it absent there, except when the murmur was very slight, whilst in this case the murmur was very loud and hissing at its point of origin.

4th. That the blood probably regurgitated into one of the auricles, or into the pulmonary artery.

5th. That it did not regurgitate into the left auricle; inasmuch as, had it done so, we should have found more decided pulmonary engorgement, and hæmoptysis rather than hæmorrhoids or hepatic enlargement.

6 th. That the opening was rather into the pulmonary artery than into the right auricle; because aneurisms more frequently perforate the former. In eighteen cases of varicose 
aneurism recorded by Dr. Thurnam, eleven had perforated the artery, and only seven other parts, even including cases of communication with the superior and inferior venæ cavæ. Of 29 cases collected by Dr. Sibson-

$\begin{array}{ccc}17 \text { opened into the pulmonary artery. } \\ 6 & \text { r } & \begin{array}{l}\text { right auricle. } \\ \text { right ventricle. }\end{array} \\ 3 & " & \text { left ventricle. } \\ 0 & " & \text { left auricle. } \\ - & & \end{array}$

In seven others the aneurism had opened into the vena cava descendans.

Further, the frémissement was to the left of the sternum, whereas in recorded cases of openings into the right auricle the fremissement presented its maximum intensity at the right of the sternum.

7 th. That the communication was probably owing to aneurismal perforation of the aorta at or near its origin. Because there was no history of cyanosis to indicate any congenital malformation of the heart. The increased vertical dulness, age, formation, and occupation of the patient, as well as some points of his history, lent strength to this view.

8th. That the aneurism sprang from the root of the aorta, or near it.

To proceed now with the narration of the case.

No change took place in the physical signs during his residence in the hospital of about three weeks, except that after a considerable hæmorrhoidal loss the murmurs and frémissement all became intensified; they, however, soon reverted to their former condition.

At his urgent request that something should be done for the piles, I allowed Mr. Wilders, our able house-surgeon, to touch them with nitric acid. After this they bled less, though the hæmorrhage was never completely suppressed, nor indeed did I wish that it should be. 
He declared that he was quite well, except that his breathing was a little short. I could not induce him to remain longer in the hospital, as he was determined to resume his employment, which he accordingly did.

Upon the morning of the 14th of June, 1860, having been at work all night, he was seized about 6 a.m. with præcordial oppression and faintness, such as he had never previously experienced. Nevertheless, after getting some brandy and lying down for a couple of hours, he was able to walk home, a distance of about a mile. After that he became rapidly worse, and was seen by Mr. Pemberton, to whom I am indebted for the further account of this case.

Mr. Pemberton says, "I found him with laborious breathing, and an intense expression of anxiety, referring his suffering to a load at the epigastrium. The hand placed over the chest in the cardiac region experienced a purring sensation, clearly and distinctly marked sounds. These were characterised by loud, continuous, doublerushing sounds audible all over the cardiac region, but more intensely so at the base of the heart and up the aorta. There was entire absence of superficial venous distension; hæmorrhage, continuous and arterial, from the hæmorrhoidal veins. The condition of the patient varied only in the occasional abatement of his feelings of oppression.

"Treatment.-Brandy, ether, and counter-irritation.

"He continued much the same until themorning of the28th of June. I visited him about 11 a.m., and found him gasping for breath. He would suddenly jump out of bed on to the floor, throwing himself at full length, and exclaiming that he was dying. He was truly so, and having informed his wife that I did not think he could live many hours, I had gone downstairs, when I heard a cry, and returned to his room. I found him lying on the edge of the bed, partly on the floor, with his head downwards, and a copious effusion of blood from the rectum. I at once raised him on to the pillow, when he gave a feeble gasp or two and expired." 
Post-mortem, twenty-four hours after death, at which, by the politeness of Mr. Pemberton, I was present.-We found congestion of both lungs, with serous effusion of no great amount in either pleura. Abundant serous effusion into the abdominal cavity. The liver much enlarged and congested. The kidneys healthy. A few ounces of serum in the pericardium. The heart enlarged, chiefly on the right side. We also discovered the aneurism; but as the specimen was subsequently examined with care by Dr. Bracey, I shall employ his description of it.

"The aneurism springs from the origin of the aorta, and projects forwards compressing the right ventricle and the commencement of the pulmonary artery. It would contain, when distended, a small hen's egg. It communicates with the aorta by a round opening, about seven lines in diameter, just above and between the right and left semilunar valves. It also communicates with the pulmonary artery by a vertical slit with regular margin and smooth, rounded edges, about three lines in extent; this is behind the posterior division of the pulmonary artery, in the corresponding sinus of Valsalva. Below this and to the right is another opening, leading into the right ventricle between and below the posterior and right valves; its margins are ragged and thin, greatest diameter (transverse) about four lines. There are a few small patches of atheroma in the aorta. Heart large; valves healthy."

I may add, that there was no coagulum in the aneurism. The edges of the slit in the right ventricle were not only ragged and thin, but also stained with blood, which was not the case with the other openings.

As to the sequence of the lesions in this patient, I suppose that the aneurism itself had existed for probably not less than six months at the time that I first saw him; for that length of time, at all events, he had been suffering from hæmorrhoids, due, it may be presumed, to the pressure of the tumour upon the pulmonary artery. Upon this supposition, we should date the communication with the pulmonary artery from the time that he made the violent effort to 
escape being run over. I cannot doubt for a moment that the perforation of the right ventricle took place on the morning of the 14th of June.

The case is of interest as being, so far as my knowledge goes, the first in which such a lesion has been discovered during life by physical means.

It is of importance as establishing - at least I am disposed to think so-the rational diagnosis of similar lesions in future.

Granting the correctness of this belief, a great advance has been made in the diagnosis of varicose aneurisms in general, since, as before mentioned, this particular species is by far the most common of the whole class.

At the same time it must be borne in mind that the key to my opinion was the non-conduction of the second murmur to the heart's apex. We should therefore be at a loss in most cases where-

1st. aortic, or

2nd. pulmonary regurgitation existed;

3rd. In which the new communication produced no murmur with the second cardiac sound;

4th. Wheretherealso existed an opening intoeither ventricle,

5 th. or if there was an opening into the left auricle or its appendix. Though we should, I think, be saved from error by attention to the physical condition of the lungs, as compared with that of the liver, \&c.; and besides, such a lesion is of the utmost rarity.

The case is both interesting and important, as showing with how little inconvenience such a lesion may exist.

Much stress has always been laid upon thrill as an indication of varicose aneurism; and this rightly, when the site of thrill is so remote from the heart as to preclude the possibility of a cardiac origin, but not otherwise, since there seems to be no limit to the amount of thrill producible by simple valvular lesions. In the present case the thrill was not employed to prove the existence of preternatural communication, though its presence lent a certain amount of strength to 
that theory. It was employed, secondarily, to indicate the exact position of this communication ; at the same time, even in its absence, the site of the second murmur would have given precisely as much as, and perhaps even more reliable information than the thrill itself, since it is by no means certain that thrill is invariably most intense at the site of the lesion upon which it depends. Neverthless, were such a thrill, to our own knowledge, suddenly developed over the heart, it would have almost as great semeiotic value as it undoubtedly has in varicose aneurisms remote from the heart.

Although I am perfectly content to rest the validity of any diagnosis in this case upon its own merits, yet the arguments upon which it was founded do gain weight from a case recorded by Professor Hughes Bennett, in his 'Principles and Practice of Medicine.' The physical signs, general history, and dissection, were nearly identical with those of my own patient. Dr. Bennett did not deduce from them any diagnosis during life; in fact, he says that there was "evidence of a profound lesion of the heart, although its nature was very mysterious, the more so as no thrill or tremor was detected. The case, however, was at once made clear, and the nature of the sounds explained, by the examination of the body after death." It is at the same time due to myself to state that I was not aware of the existence of Professor Bennett's record, until after the death of my patient. 